\title{
Treponema Infections and Orofacial Involvement
}

\author{
Bhattacharyya Satarupa Ghosal ${ }^{1}$ and Bhattacharyya Sayan ${ }^{2 *}$ \\ ${ }^{1}$ Dental Surgeon, India \\ ${ }^{2}$ Assistant Professor, Department of Microbiology, AIIH\&PH, India
}

*Corresponding author: Bhattacharyya Sayan, Assistant Professor, Department of Microbiology, AIIH\&PH, Kolkata, India

\begin{abstract}
Teeth, gum, and palate may be affected by different congenital and acquired infections caused by Treponema spp. These are somewhat rarely found now but still very important to know for diagnostic as well as epidemiological purposes. Some of these pathogens are generally transmitted and some by close skin to sin contact. Orofacial involvement may be found in various stages of these infections. In this review, the authors have tried to compile available scientific information about these infections affecting the orofacial region.
\end{abstract}

Keywords: Treponema; congenital; acquired; teeth; Gumma; Gangosa

\section{Introduction}

Treponema spp. are spirochaetes or spiral bacteria that cause congenital infections as well as sexually and non-sexually transmitted infections. Many a times they cause involvement of the orofacial region. These issues are discussed less often, and hence the authors have tried to review available scientific information about his.

\section{Material and Methods}

Systematic review of the available articles and book chapter were done for preparing this review article.

\section{Congenital syphilis}

Late congenital syphilis in the child or adolescent corroborates with tertiary syphilis in the adult, although the cardiovascular system is seldom involved. Typical manifestations comprise bony and dental abnormalities like skull bossing and Hutchinson's teeth and inflammatory lesions of the cornea (interstitial keratitis) and joints (Clutton's joints) [1]. Syphilitic vasculitis at or during the time of birth can damage the developing tooth buds and lead to various dental anomalies. Hutchinson teeth are abnormal permanent upper central incisors which are peg-shaped and notched, generally with obvious thinning and discoloration of enamel in the area of the notch [2]. They are widely spaced and usually shorter than the lateral incisors, and the width of the biting surface is less than that of the gingival margin [2]. Mulberry molars are also found in congenital syphilis. These mulberry molars (also known as Moon or Fournier molars) are multicuspid first molars in which the tooth's grinding surface, which is narrower than that at the gingival margin, has multiple small cusps instead of the usually seen four well-formed cusps [2].

\section{Primary syphilis}

It has been reported that Syphilitic chancre can develop in the mouth, lip or tongue in some cases, depending on the aberrant sexual practices [3]. Oral manifestations are observed in approximately $4-12 \%$ of Syphilitic patients, reflecting the sexual practices $[3,4]$. These lesions generally present as painless indurated ulcers on the tongue, gingiva, soft palate, or lips, that last for 3 to 7 weeks and are associated with lymphadenopathy involving the submandibular and cervical regions [5].

\section{Secondary syphilis}

In secondary stage of Syphilis, large, raised sores and rashes on mucous membranes, such as gums or tongue may be found [4]. Pharyngitis is very commonly found in these cases, and when it affects the oral cavity, secondary Syphilis appear as oval 
reddish maculae or maculopapular eruptions called syphilitic rrosette [6]. In some cases, lesions looking like condyloma latum may also occur in the oral cavity, with the labial commissure as the preferred site of involvement [6]. Ulceronodular disease or "Lues maligna", also called malignant Syphilisis an aggressive generalized form of secondary syphilis which presents with fever, headache, and myalgia, followed by a papulopustular eruption that quickly converts itself into necrotic, sharply demarcated ulcers with hemorrhagic brown crusts [7]. It is seen usually in immunocompromised hosts but rarely also in immunocompetent hosts. These crusts are organized in rupioid layers, usually on the face and scalp. The mucosa is involved in about one third of affected patients [7]. Lues maligna can cause crateriform or shallow ulcers on the gingivae, palate or buccal mucosa, with multiple erosions on the hard and soft palates, tongue and lower lip [7]. Other atypical or non-specific lesion may be found in secondary syphilis, like aphthous ulcers or other painful or painless ulcers [8].

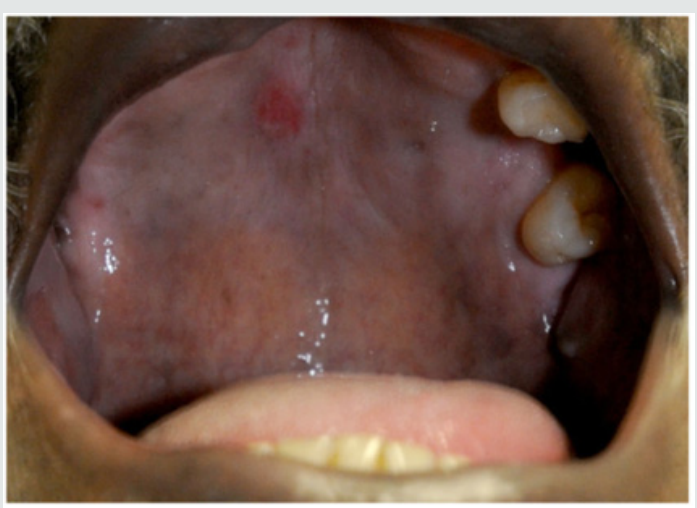

Figure 1: Primary Sypghilis affecting the mouth (palate).

\section{Gumma in syphilis and orofacial affection}

Gumma is typically found in tertiary Syphilis. Gummas tend to arise on the hard palate and tongue, although very rarely they may also occur on the soft palate, lower alveolus, and parotid gland [7]. Oronasal perforation can also occur. Rarely, a gumma lesion may even invade blood vessels, like the inferior alveolar artery [7]. Ulcers heal generally, but especially in tongue, residual fissuring can occur.

\section{Risk of acquiring HIV in Oral Syphilis}

Oral syphilis mainly influences HIV disease by increasing the chances transmission of HIV and other related viruses by oral sexual routes [7]. Oral ulcerative disease, like that seen in all the stages of Syphilis, will increase the HIV load in the mouth, and hence the increased potential for HIV transmission via oral sex [7].

\section{Association with malignancy}

Syphilitic leukoplakia appears as a homogenous white patch affecting large areas of the dorsum of the tongue. There are few reports of syphilitic leukoplakia, but it is unclear whether this lesion truly reflects syphilis or more often a tobacco smoking habit [7]. However, there is controversy regarding whether risk of oral squamous cell carcinoma in syphilis is a direct consequence of infection or is actually the combined effect of known causative factors for oral malignancy, such as tobacco smoking, alcohol, and malnutrition [7].

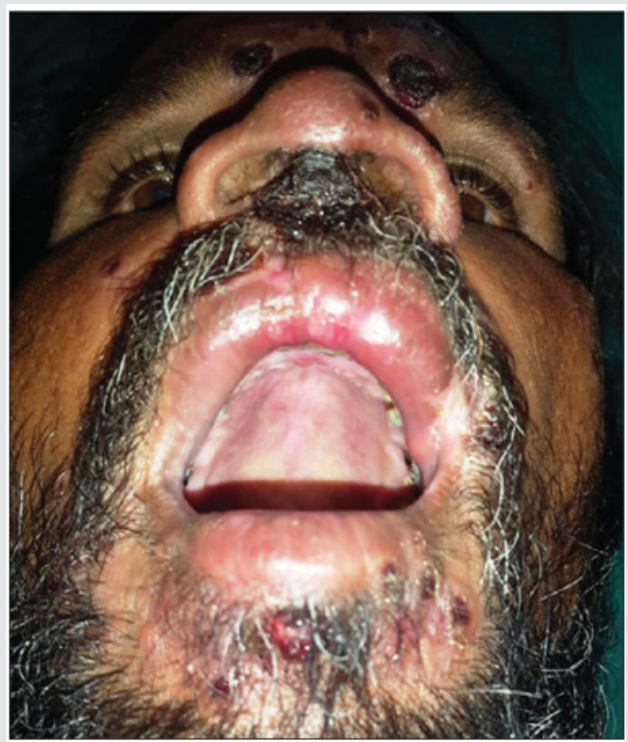

Figure 2: Lues maligna in Mouth.

\section{Endemic treponematoses and orofacial involvement}

Endemic Treponema infections can also cause oral mucosal and orolabial involvement. Details are discussed below.

\section{Yaws and affection of teeth and palate}

Tertiary lesions, or "late yaws", are associated with soft tissue destruction in $10 \%$ of cases 2 leading to deep ulceration, gangosa (destruction of the maxilla), hyperkeratotic palmar and plantar lesions, and bony involvement These lesions typically present 5 years following initial presentation of early yaws. However, this stage is not infectious [9]. Goundou as a complication of Yaws can cause periosteitis of the maxilla and bilateral pernasal swelling. This is very rarely seen now. Gangosa is a Gumma-like progressive necrotic orofacial lesion that can cause oronasal perforation.

\section{Bejel}

Bejel is found in Africa and Middle East. The onset of bejel usually occurs in childhood and typically presents with a mucous 
patch on the buccal mucosa. Mucosal lesions are followed by papulosquamous and erosive papular lesions of the trunk and extremities. Latency can occur if the early stages of the disease are not treated [10].

\section{Involvement of the so-called commensal flora}

Commensal bacterial flora of the orofacial region also plays a role in periodontal disease. Patients are at high risk of developing periodontal diseases if bacteria like Porphyromonas gingivalis, Tannerella forsythia, and Treponema denticola are in the subgingival plaque [11]. Other normal flora poses a lesser risk of these infections. T. denticola has been established as a risk factor fora endodontic disease as well as periodontal disease, and already its association with orofacial abscesses and periapical radiolucencies are known [12]. Monoinfection with T. denticola can cause aggressive disease and bone resorption too [12]. This also depends on the load or count of T. denticola in the mouth. Root canal infection with T. denticola and other oral spirochetes may also cause dissemination to important target organs, particularly in immunosuppressed hosts [12].

\section{Future directions}

Orofacial affection by Treponemes is thus a very interesting area of research. However not much information is available in this aspect. More research is awaited in this area.

\section{Discussion}

Different Treponema species can infect orofacial structures and are thus very important. Different stages of infection by these bacteria can cause orofacial affection. These are particularly common in different stages of Syphilis. In spite of the advances in medicine toward the prevention and early diagnosis and treatment of syphilis, this disease still remains a public health problem throughout the world [8]. Some might also cause premalignant lesions. Hence clinicians need to be vigilant, and we should look for these cases since they are often misdiagnosed and confused with other similar lesions. Other lesions like those in skin and bones can be found in these diseases. Oral syphilitic infection, hence, must be considered if and when a patient presents with oral lesions and also skin manifestations of secondary disease, even without any genital ulcerations [5].

\section{Conclusion}

Information about Treponemes affecting the orofacial region is scarce and more this topic merits more research and scientific attention.

\section{References}

1. Richens J, Mayaud P, Mabet DCW (2014) Sexually Transmitted infections (excluding HIV). Manson's Tropical Infectious Diseases (Twenty-Third Edition) pp. 292-318.

2. Kollmann TR, Dobson S (2011) in Infectious Diseases of the Fetus and Newborn $\left(7^{\text {th }}\right.$ Edition).

3. What Causes Oral Syphilis and How Do You Treat It.

4. Ficarra G, Carlos R (2009) Syphilis: the renaissance of an old disease with Oral implications. Head Neck Pathol 3(3): 195-206.

5. Streight KL, Paranal RM, Musher DM (2019) The oral manifestations of syphilitic disease: a case report. J Med Case Reports 13: 227.

6. de Andrade RS, de Freitas EM, Rocha BA, Gusmão ES, Filho MR, et al. (2018) Oral findings in secondary syphilis. Med Oral Patol Oral Cir Bucal 23(2): 138-143.

7. Leão Jair Carneiro, Gueiros Luiz Alcino, Porter, Stephen R (2006) Oral manifestations of syphilis. Clinics 61(2): 161-166.

8. Seibt CE, Munerato MC (2016) Secondary syphilis in the oral cavity and the role of the dental surgeon in STD prevention, diagnosis, and treatment: a case series study. Brazilian J INfec Di 20(4): 393-398.

9. Santos MA, Faldetta KF, Zaenglein AL (2015) Yaws: Rebound of a forgotten disease. Glob Dermatol.

10. Whitcup SM (2010) Spirochetal Diseases. Uveitis. $4^{\text {th }}$ Edition pp. 142160.

11. Infections of the teeth, gingivae, and jaws.

12. Foschi F, Izard J, Sasaki H (2006) Treponema denticola in disseminating endodontic infections. J Dent Res 85(8): 761-765.
(C) (P) This work is licensed under Creative Commons Attribution 4.0 License

To Submit Your Article Click Here: Submit Article

DOI: $10.32474 /$ IPDOAJ.2021.05.000213

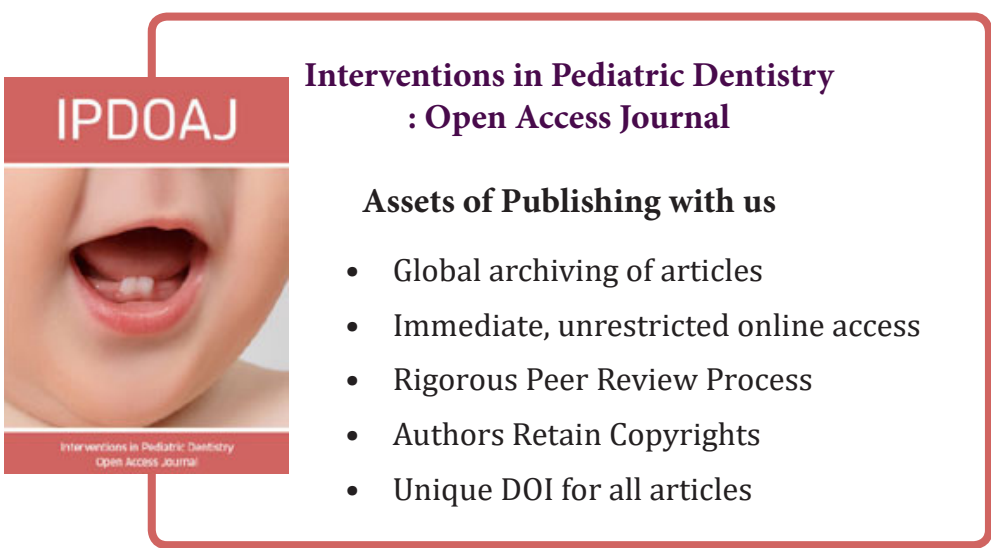

